\title{
Interplay between Zhang-Rice singlets and high-spin states in a model for doped $\mathrm{NiO}_{2}$ planes
}

\author{
Tharathep Plienbumrung $\odot,,^{1,2}$ Maria Daghofer $\odot,{ }^{1,2}$ and Andrzej M. Oleś $\circledast^{3,4, *}$ \\ ${ }^{1}$ Institute for Functional Matter and Quantum Technologies, University of Stuttgart, Pfaffenwaldring 57, D-70550 Stuttgart, Germany \\ ${ }^{2}$ Center for Integrated Quantum Science and Technology, University of Stuttgart, Pfaffenwaldring 57, D-70550 Stuttgart, Germany \\ ${ }^{3}$ Max Planck Institute for Solid State Research, Heisenbergstrasse 1, D-70569 Stuttgart, Germany \\ ${ }^{4}$ Institute of Theoretical Physics, Jagiellonian University, Profesora Stanistawa Łojasiewicza 11, PL-30348 Kraków, Poland
}

(Received 18 December 2020; accepted 8 March 2021; published 22 March 2021)

\begin{abstract}
Superconductivity found in doped $\mathrm{NdNiO}_{2}$ is puzzling as two local symmetries of doped $\mathrm{NiO}_{2}$ layers compete, with presumably far-reaching implications for the involved mechanism: A cupratelike regime with Zhang-Rice singlets is replaced by local triplet states at realistic values of charge-transfer energy, which would suggest a rather different superconductivity scenario from high- $T_{c}$ cuprates. We address this competition by investigating $\mathrm{Ni}_{4} \mathrm{O}_{8}$ clusters with periodic boundary conditions in the parameter range relevant for the superconducting nickelates. With increasing value of charge-transfer energy we observe upon hole doping the expected crossover from the cuprate regime dominated by Zhang-Rice singlets to the local triplet states. We find that smaller charge-transfer energy $\Delta$ is able to drive this change in the ground-state character when realistic values for nickel-oxygen repulsion $U_{d p}$ are taken into account. For large values of the charge-transfer energy, oxygen orbitals are less important than in superconducting cuprates as their spectral weight is found only at rather high excitation energies. However, a second $\mathrm{Ni}(3 d)$ orbital can easily become relevant, with either the $x y$ or the $3 z^{2}-r^{2}$ orbitals contributing in addition to the $x^{2}-y^{2}$ orbital to the formation of triplet states. In addition, our result that $U_{d p}$ (acting between $\mathrm{Ni}$ and $\mathrm{O}$ ) favors on-site triplets implies that correlation effects beyond purely on-site interactions should be taken into account when obtaining effective two-band models.
\end{abstract}

DOI: 10.1103/PhysRevB.103.104513

\section{INTRODUCTION}

Two-dimensional (2D) nickelates such as $\mathrm{LaNiO}_{2}$ were theoretically proposed long ago [1] as candidate materials for unconventional superconductors, but only recently has superconductivity been found in $\mathrm{Sr}-$ doped $\mathrm{NdNiO}_{2}$ thin films [2]. This discovery could be classified as fulfilling the paradigm of high- $T_{c}$ superconductivity in systems similar to cuprates where both $e_{g}$ symmetries contribute at the Fermi surface [3]. Indeed, nickelate heterostructures were considered to be the most promising [4,5], but it took another decade until superconductivity was found in quasi-2D films [2]. Shortly after the discovery of superconductivity in doped $\mathrm{NdNiO}_{2}$, the search for its mechanism began, and it became clear that once again we are dealing with unconventional superconducting (SC) materials. However, the situation in the theory is somewhat similar to SC cuprates, where after their discovery in 1986 [6], the mechanism of high- $T_{c}$ superconductivity still remains puzzling $[7,8]$.

Recently, several groups calculated the electronic structure and tried to understand the essential differences from cuprate physics. Two remarkable differences between the $\mathrm{CuO}_{2}$ planes of correlated insulator $\mathrm{La}_{2} \mathrm{CuO}_{4}$ and the $\mathrm{NiO}_{2}$

\footnotetext{
*a.m.oles@fkf.mpi.de
}

Published by the American Physical Society under the terms of the Creative Commons Attribution 4.0 International license. Further distribution of this work must maintain attribution to the author(s) and the published article's title, journal citation, and DOI. Open access publication funded by the Max Planck Society. planes in $\mathrm{NdNiO}_{2}$ became clearly evident. First, the nickelates are "self-doped" due to the their rare-earth bands, so that an "undoped" compound does not correspond to an undoped $\mathrm{NiO}_{2}$ layer $[9,10]$. Regardless of the level of self-doping realized in superconducting $\mathrm{NdNiO}_{2}$, we focus here on understanding the undoped $\mathrm{NiO}_{2}$ layers, which we regard as a kind of idealized "parent compound." The argument for studying a $\mathrm{NiO}_{2}$ plane is a certain effective decomposition into a threedimensional band including rare-earth states and a more 2D band of $x^{2}-y^{2}$ character [11]. Second, the $\mathrm{NiO}_{2}$ layers miss the apical oxygen ions present in the $\mathrm{CuO}_{2}$ layers. However, the $\mathrm{NiO}_{2}$ plane of the infinite $2 \mathrm{D}$ layer in $\mathrm{NdNiO}_{2}$ is similar to the $\mathrm{CuO}_{2}$ plane of $\mathrm{CaCuO}_{2}$, where the apical oxygens are missing as well. One might argue that the properties of these planes would then be similar, but they in fact reflect two different parameter regimes of the charge-transfer model which describes them both.

In spite of great similarity between $\mathrm{CuO}_{2}$ and $\mathrm{NiO}_{2}$ planes, there are thus substantial differences in the electronic structure. While $\mathrm{La}_{2} \mathrm{CuO}_{4}$ is a charge-transfer system, the $\mathrm{NiO}_{2}$ planes in $\mathrm{NdNiO}_{2}$ have large charge-transfer energy $\Delta$, which indicates more a Mott-Hubbard system [12]. This is also confirmed in other works [9,13-18], and thus Ni-O hybridization plays here a less important role than in cuprates. Arguments were also given that the electronic structure in nickelate superconductors could be reduced to a one-band Hubbard model [19]. The undoped system has one hole in the $x^{2}-y^{2}$ Ni orbital which is the $\left|b_{1}\right\rangle$ symmetry state, but Zhang-Rice (ZR) singlets $(S=0)\left|b_{1} L_{b_{1}}\right\rangle$ are much weaker in doped systems. These states compete with high-spin $(S=1)\left|a_{1} b_{1}\right\rangle$ states when doping increases [12]. 
However, perhaps the most important difference between the above two classes of materials is that there is only one band which crosses the Fermi level in cuprates, while two bands cross the Fermi level in nickelates $[3,17,20,21]$. This suggests that indeed both $e_{g}$ orbitals could contribute at finite $\mathrm{Sr}$ doping to the properties of $\mathrm{NdNiO}_{2}$. In the impurity model one finds therefore a transition from the singlet to triplet local configuration at the doped $\mathrm{Ni}^{2+}$ ion [12], and we shall investigate here how this changes by going to a more extended $\mathrm{Ni}-\mathrm{O}$ system than a single $\mathrm{NiO}_{4}$ unit, i.e., beyond the impurity model. Furthermore, we show below that the transition to the regime where high-spin states contribute depends on the value of intersite Coulomb repulsion $U_{d p}$.

Another question which we want to address here is the nature of high-spin states in doped materials. In a cuprate model the orbital which is close to the top $x^{2}-y^{2}$ orbital is the second $e_{g}$ state of $3 z^{2}-r^{2}$ symmetry (called also $z^{2}$ below), and $S=1$ states could form by hole doping [22]. They would compete with ZR singlets [23]. Recent electronic structure results for $(\mathrm{Nd}, \mathrm{Sr}) \mathrm{NiO}_{2}$ found using a combination of dynamical mean-field theory of correlated electrons and band structure methods indicate a remarkable orbital-selective renormalization of the $\mathrm{Ni} 3 d$ bands [24]. However, for a $\mathrm{NiO}_{4}$ plaque in $\mathrm{NdNiO}_{4}$, the crystal fields are quite different, and the orbital $x y$ is the first orbital below the $x^{2}-y^{2}$ orbital occupied by one hole, while the $z^{2}$ orbital is at the bottom of the orbital states [25]. This sequence of the orbital states at a Ni ion was recently confirmed by quantum-chemistry calculations [26]. It is thus challenging to ask to which $3 d$ orbital doped holes will go in a doped material and whether this could have any physical consequences.

The paper is organized as follows. In Sec. II we explain the charge-transfer model and its parameters. The methods to analyze finite systems, i.e., exact diagonalization and the variational cluster approximation (VCA), are introduced in Sec. III. The numerical results are presented and discussed in Sec. IV. The paper is summarized in Sec. V. In the Appendix we analyze briefly the consequences of the partial filling of the $x y$ orbital on $\mathrm{Ni}$ ions and conclude that the general conclusions concerning the possibility of the participation of high-spin states in the ground state are not affected.

\section{THE CHARGE-TRANSFER MODEL}

We introduce the multiband $d-p$ Hamiltonian for a $\mathrm{NiO}_{2}$ plane where we consider the 2D $2 \times 2$ cluster shown in Fig. 1 (with periodic boundary conditions), which includes four orbitals per $\mathrm{NiO}_{2}$ unit cell: two $e_{g}$ orbitals $\left\{3 z^{2}-r^{2}, x^{2}-y^{2}\right\}$ at each $\mathrm{Ni}^{+}$ion and one bonding $2 p_{\sigma}$ orbital (either $2 p_{x}$ or $2 p_{y}$ ) at each oxygen ion in the $2 \mathrm{D}$ plane (12 is the total number of ions in the periodic $\mathrm{Ni}_{4} \mathrm{O}_{8}$ cluster),

$$
\mathcal{H}=H_{d p}+H_{p p}+H_{\text {diag }}+H_{\text {int }}^{d}+H_{\text {int }}^{p} .
$$

Here, the first two terms in the Hamiltonian (1) stand for the kinetic energy: $H_{d p}$ includes the $d-p$ hybridization $\propto t_{p d}$, and $H_{p p}$ includes the interoxygen $p-p$ hopping $\propto t_{p p}$,

$$
H_{d p}=\sum_{\{m \alpha ; j v\}, \sigma}\left(t_{m \alpha ; j v} \hat{d}_{m \alpha, \sigma}^{\dagger} \hat{p}_{j v, \sigma}+\text { H.c. }\right) \text {, }
$$

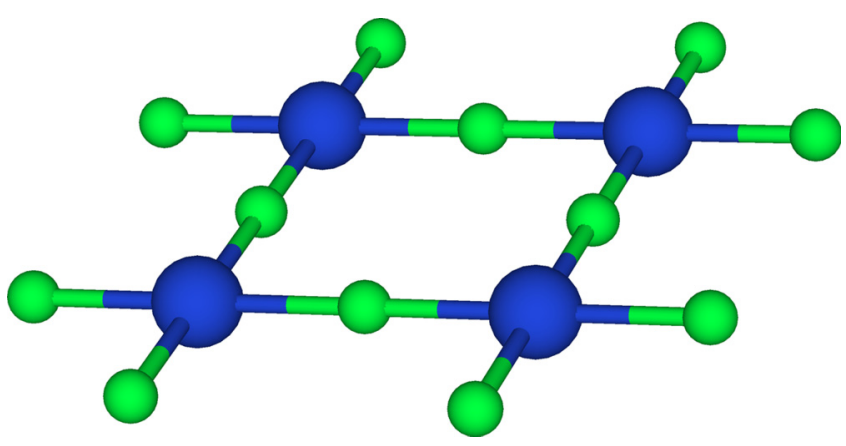

FIG. 1. Schematic picture of the 2D periodic cluster $\left(\mathrm{NiO}_{2}\right)_{4}$ used here to model the $\mathrm{NiO}_{2}$ infinite plane. Blue and green balls stand for $\mathrm{Ni}$ and $\mathrm{O}$ ions in the plane.

$$
H_{p p}=\sum_{\{i \mu ; j v\}, \sigma}\left(t_{i \mu ; j v} \hat{p}_{i \mu, \sigma}^{\dagger} \hat{p}_{j v, \sigma}+\text { H.c. }\right)
$$

where $\hat{d}_{m \alpha, \sigma}^{\dagger}\left(\hat{p}_{j,, \sigma}^{\dagger}\right)$ is the creation operator of an electron at nickel site $m$ (oxygen site $i$ ) in an orbital $\alpha$. At Ni ions, $\alpha \in\{z, \bar{z}\}$, where $z$ and $\bar{z}$ stand for $3 z^{2}-r^{2}$ and $x^{2}-y^{2}$ orbitals, while at $\mathrm{O}$ ions, $v \in\{x, y\}$, where $x$ and $y$ stand for $p_{x}$ and $p_{y}$ orbitals, with up or down spin, $\sigma \in\{\uparrow, \downarrow\}$. The model includes two $3 d$ orbital states of $e_{g}$ symmetry at $\mathrm{Ni}^{+}$ ions and one $2 p_{\sigma}$ bonding oxygen orbital state at oxygen ions in the $(a, b)$ plane, either $2 p_{x}$ for the Ni-O bond $\langle m i\rangle \| a$ or $2 p_{y}$ for the bond $\langle m i\rangle \| b$. We study an isolated $\mathrm{NiO}_{2}$ plane in $\mathrm{NdNiO}_{2}$ and so do not consider $2 p_{z}$ orbitals at apical oxygen positions.

In the following we will use shorthand notation, and instead of $\left\{x^{2}-y^{2}, 3 z^{2}-r^{2}\right\}$ we shall write $\{(\bar{z}),(z)\}$ - this emphasizes the fact that the $z$ axis is chosen as the quantization axis for this $e_{g}$ orbital basis, while "( )" bracketing is used here to distinguish two $\mathrm{Ni}(3 d)$ orbitals from $\mathrm{O}\left(2 p_{\sigma}\right)$ orbitals, labeled as $\{x, y\}$. The elements of the matrices $\left\{t_{m \alpha ; j v}\right\}$ and $\left\{t_{i \mu ; j v}\right\}$ are assumed to be nonzero only for nearestneighbor nickel-oxygen $d-p$ pairs and for nearest-neighbor oxygen-oxygen $p-p$ pairs. The next-nearest-neighbor hopping is neglected. (The nonzero $\left\{t_{m \alpha ; j v}\right\}$ and $\left\{t_{i \mu ; j v}\right\}$ elements are standard and are listed, e.g., in the Appendix of Ref. [27]).

The one-particle (level) energies are included in $H_{\text {diag }}$, where the on-site elements of kinetic energy are bare level energies and local crystal fields. The cluster geometry and precise forms of different terms are standard; these terms were introduced in the previous realizations of the three-band $d-p$ model for $\mathrm{CuO}_{2}$ planes [28] and for other transition-metal oxides [27,29]. The diagonal part $H_{\text {diag }}$ depends on electron number operators. It takes into account the effects of local crystal fields and the difference of reference orbital energies (here we employ the hole notation, $\Delta>0$ ),

$$
\Delta=\varepsilon_{d}-\varepsilon_{p},
$$

between $d$ and $p$ orbitals (for bare orbital energies), where $\varepsilon_{d}$ is the average energy of all $3 d$ orbitals, i.e., the reference energy before they split in the crystal field due to the surrounding oxygens. We fix this reference energy for all $3 d$ orbitals to 
zero, $\varepsilon_{d}=0$, and use only $\Delta=-\varepsilon_{p}$, and thus we write

$$
H_{\text {diag }}=\Delta \sum_{i ; \mu=x, y, z ; \sigma} \hat{p}_{i \mu, \sigma}^{\dagger} \hat{p}_{i \mu, \sigma}+\Delta_{z^{2}} \sum_{m \sigma} \hat{n}_{m(z) \sigma} .
$$

The first sum is restricted to oxygen sites $\{i\}$, while the second one runs over nickel sites $\{m\}$. The crystal-field splitting strength vector $\left(\Delta_{z^{2}}\right)$ describes the splitting between two $e_{g}$ orbitals; here, we assume $\Delta_{z^{2}}=0$ to find the lowest value of $\Delta$ for which the ground state of the $\mathrm{NiO}_{2}$ plane changes. A finite value of $\Delta_{z^{2}}=2.0 \mathrm{eV}$ is assumed in the Appendix. Note that the charge-transfer energy $\Delta$ in Eq. (4) is defined for bare levels.

In Eq. (1), $H_{\text {int }}^{d}$ and $H_{\text {int }}^{p}$ stand for the intra-atomic Coulomb interactions at $\mathrm{Ni}$ and $\mathrm{O}$ ions, respectively. Local interactions at nickel ions, $\mathcal{H}_{\text {int }}^{d}$, are rotationally invariant in the orbital space [30] and depend on three Racah parameters [31]. For systems where only one kind of orbital is involved, two Kanamori parameters suffice: (i) intraorbital Coulomb interaction $U$ and (ii) Hund's exchange $J_{H}$. Here, for a pair of $e_{g}$ electrons in different orbitals one finds $J_{H}=4 B+C$ (a similar expression can also be written for $t_{2 g}$ electrons),

$$
\begin{aligned}
\mathcal{H}_{\text {int }}^{d}= & U_{d} \sum_{i \alpha} \hat{n}_{i \alpha \uparrow} \hat{n}_{i \alpha \downarrow}+J_{H} \sum_{i, \alpha \neq \beta} \hat{d}_{i \alpha \uparrow}^{\dagger} \hat{d}_{i \alpha \downarrow}^{\dagger} \hat{d}_{i \beta \downarrow} \hat{d}_{i \beta \uparrow} \\
& +\sum_{i}\left[\left(U_{d}-\frac{5}{2} J_{H}\right) \hat{n}_{i(z)} \hat{n}_{i(\bar{z})}-2 J_{H} \hat{\bar{S}}_{i(z)}^{d} \cdot \hat{\vec{S}}_{i(\bar{z})}^{d}\right] .
\end{aligned}
$$

Interorbital Coulomb interactions $\propto \hat{n}_{i \alpha} \hat{n}_{i \beta}$ are expressed in terms of orbital electron density operators for a pair $\alpha<\beta, \hat{n}_{i \alpha}=\sum_{\sigma} \hat{n}_{i \alpha \sigma}=\sum_{\sigma} \hat{d}_{i \alpha \sigma}^{\dagger} \hat{d}_{i \alpha \sigma}$. Orbital spin operators $\hat{\vec{S}}_{i \alpha} \equiv\left\{\hat{S}_{i \alpha}^{x}, \hat{S}_{i \alpha}^{y}, \hat{S}_{i \alpha}^{z}\right\}$ appear in the Hund's exchange term, $-2 J_{H} \hat{\vec{S}}_{i(z)}^{d} \cdot \hat{\vec{S}}_{i(\bar{z})}^{d}$. In a Mott insulator, charge fluctuations are quenched and electrons localize due to the large energy of the lowest multiplet state, $\left(U-3 J_{H}\right) \gg t$, associated with highspin charge excitation and determining the fundamental Mott gap. For the present $\mathrm{Ni}^{+}$ions, Hund's exchange $J_{H}$ stabilizes high-spin excited states of two holes per site, with spin $S=1$.

Local interactions at oxygen ions, $\mathcal{H}_{\text {int }}^{p}$, are again rotationally invariant in the orbital space [30] and depend on two Kanamori parameters: (i) intraorbital Coulomb interaction $U_{p}$ and (ii) Hund's exchange $J_{p}$ between a pair of $2 p$ electrons in different orbitals,

$$
\begin{aligned}
\mathcal{H}_{\text {int }}^{p}= & U_{p} \sum_{i \mu} \hat{n}_{i \mu \uparrow}^{p} \hat{n}_{i \mu \downarrow}^{p}+J_{H}^{p} \sum_{i, \mu \neq \nu} \hat{p}_{i \mu \uparrow}^{\dagger} \hat{p}_{i \mu \downarrow}^{\dagger} \hat{p}_{i \nu \downarrow} \hat{p}_{i \nu \uparrow} \\
& +\sum_{i, \mu<\nu}\left[\left(U_{p}-\frac{5}{2} J_{H}^{p}\right) \hat{n}_{i \mu}^{p} \hat{n}_{i \nu}^{p}-2 J_{H}^{p} \hat{\vec{S}}_{i \mu}^{p} \cdot \hat{\vec{S}}_{i \nu}^{p}\right] .
\end{aligned}
$$

Interorbital Coulomb interactions $\propto \hat{n}_{i \mu}^{p} \hat{n}_{i v}^{p}$ are expressed in terms of orbital electron density operators for a pair $\mu<v$, $\hat{n}_{i \mu}^{p}=\sum_{\sigma} \hat{n}_{i \mu \sigma}^{p}=\sum_{\sigma} \hat{p}_{i \mu \sigma}^{\dagger} \hat{p}_{i \mu \sigma}$. Orbital spin operators, $\hat{\vec{S}}_{i \mu}^{p} \equiv\left\{\hat{S}_{i \mu}^{p x}, \hat{S}_{i \mu}^{p y}, \hat{S}_{i \mu}^{p z}\right\}$, appear in the Hund's exchange term, $-2 J_{p} \hat{\vec{S}}_{i \mu}^{p} \cdot \hat{\vec{S}}_{i v}^{p}$. In a Mott insulator, charge fluctuations are quenched and electrons localize due to the large energy of the fundamental Mott gap, $\left(U_{p}-3 J_{H}^{p}\right) \gg t$, associated with high-spin charge excitation of $p^{2}$ in the case of $p^{1}$ (or $p^{4}$ in the case of $p^{5}$ ) in the ground-state configuration. Hund's
TABLE I. Parameters of the multiband model (1) (all in eV) used in exact diagonalization calculations to model a $\mathrm{NiO}_{2}$ plane. For the hopping integrals we adopt the values from Refs. [32,33] and include oxygen-oxygen hopping elements in $H_{p p}$ given by $(p p \sigma)=0.6 \mathrm{eV}$ (here we use the Slater notation [34]). The $t_{2 g}$ and $e_{g}$ orbital states are arbitrarily taken as degenerate.

\begin{tabular}{lcccccccc}
\hline \hline & $(p d \sigma)$ & $t_{p p}$ & $\Delta$ & $U_{d}$ & $J_{\mathrm{H}}$ & $U_{p}$ & $J_{\mathrm{H}}^{p}$ & $U_{d p}$ \\
\hline Ref. [12] & 1.5 & 0.55 & 7.0 & 8.34 & 1.18 & 4.4 & 0.8 & 0.0 \\
This paper & 1.3 & 0.55 & 7.0 & 8.34 & 1.18 & 4.4 & 0.8 & $0.0-1.0$ \\
\hline \hline
\end{tabular}

exchange $J_{H}^{p}$ stabilizes, then, high-spin $S=1$ states at oxygen sites with two holes.

The parameters of the charge-transfer model Eq. (1) used in this paper are given in Table I. They are compared with the parameters used in Ref. [12]. The only difference is the somewhat smaller $(p d \sigma)=1.3 \mathrm{eV}$ hybridization element, which we consider to be more realistic; see Table I.

\section{NUMERICAL METHODS}

\section{A. Exact diagonalization}

We start with the impurity model and investigate the ground state of the $\left(\mathrm{NiO}_{4}\right)^{6-}$ cluster [12], which corresponds to the $\left(\mathrm{NiO}_{2}^{3-}\right)$ unit in the $\mathrm{NiO}_{2}$ plane doped by one hole. This cluster has a small basis of orbital states $\{(z),(\bar{z}), x, y\}$ and is diagonalized exactly for $N_{\uparrow}=N_{\downarrow}=1$ holes, i.e., one $\uparrow$-spin and one $\downarrow$-spin hole. Next we calculate the ground states of the model (1) for the $\left(\mathrm{NiO}_{2}\right)_{4}$ cluster with periodic boundary conditions using the Lanczos algorithm [35] for the orbital basis $\{(z),(\bar{z}), x, y\}$ in each unit cell. We start with the undoped system, i.e., four holes in this cluster, in the $\left(N_{\uparrow}=N_{\downarrow}=2\right)$-hole configuration. Doped systems contain $N_{\uparrow}=3, N_{\downarrow}=2$, and $N_{\uparrow}=N_{\downarrow}=3$ holes, respectively.

\section{B. Variational cluster approach}

We complement our exact diagonalization study by using the variational cluster approach (VCA) [36], which embeds the four-unit-cell cluster into a larger system. This gives us some effective access to larger lattices, e.g., momentumresolved one-particle spectral densities or density of states. We use (Lanczos) exact diagonalization to obtain the Green's function of a cluster $G_{\mathrm{Cl}}$, consisting of four nickel and six oxygen ions. The cluster self-energy $\Sigma_{\mathrm{Cl}}$ is then extracted from $G_{\mathrm{Cl}}$ and inserted into the one-particle Green's function $G$ of the thermodynamic limit. The approximation consists of replacing the (unknown) thermodynamic-limit self-energy by that of the cluster, i.e., by setting $\Sigma \equiv \Sigma_{\mathrm{Cl}}$.

According to the self-energy functional theory [37], the optimal cluster self-energy is the one optimizing the thermodynamic grand potential,

$$
\Omega=\Omega_{\mathrm{Cl}}+\operatorname{Tr} \ln G-\operatorname{Tr} \ln G_{\mathrm{Cl}} .
$$

The parameters $\left\{\vec{\tau}^{\prime}\right\}$ that can be varied to find optimized $\Sigma_{\vec{\tau}^{\prime}}$ are any one-particle parameters used when solving the small cluster. We focus here on an overall fictitious chemical potential $\mu^{\prime}$ to ensure thermodynamic consistency [38] as well as a staggered antiferromagnetic field $h^{\prime}$ favoring Néel 

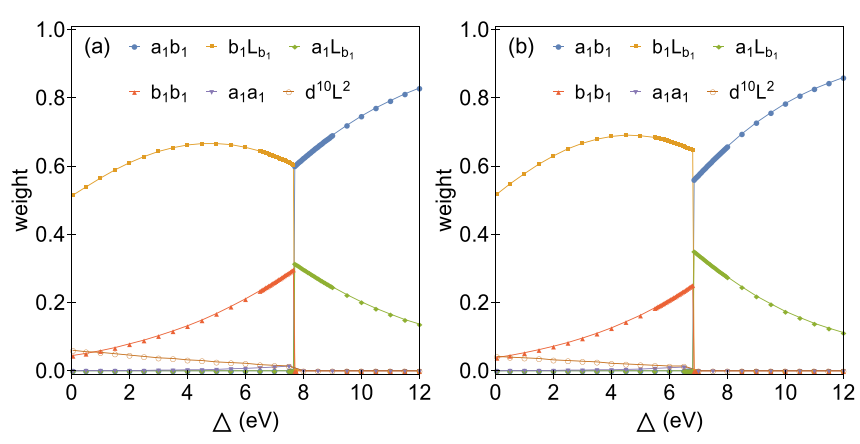

FIG. 2. Weight distribution of different components in a single $\mathrm{NiO}_{2}^{2-}$ cluster of a doped $\mathrm{NiO}_{2}$ plane, with $N_{\uparrow}=N_{\downarrow}=1$ hole, for two parameter sets of Table I. (a) Our calculation reproduces qualitatively the transition between two ground-state symmetries as in Fig. 4 in Ref. [12]; the transition is seen at somewhat lower $\Delta \simeq 7.7 \mathrm{eV}$. (b) Somewhat enhanced triplet symmetry for finite $U_{d p}=1 \mathrm{eV}$.

order. Neither has been seen to significantly affect the results reported here.

Static observables such as spin or orbital densities as well as dynamic quantities such as the one-particle density of states of k-resolved one-particle spectra are then obtained from the approximated Green's function $G$. The kinetic energy is thus fully included while interaction effects are truncated to those accessible to the directly solved cluster. A slight difficulty lies in the Coulomb repulsion $U_{p d}$ between $\mathrm{Ni}$ and $\mathrm{O}$ ions, as this can connect orbitals inside the cluster with others outside. In contrast to intercluster hopping, such intercluster interactions cannot rigorously be included in the VCA, but have to be truncated or approximated. We have here allowed periodic boundary conditions for these interactions. While this would likely be too crude an approximation if intersite interactions are close to driving an ordered phase (e.g., a charge density wave) [39-41], it has been shown to be good enough to stabilize uniform charges in the study of a strongly related three-band model for cuprates [42]. Since the oxygen ions have lower occupation in this paper, the approximation can here be expected to be even less critical.

\section{GROUND STATE AT INCREASING $\Delta$}

\section{A. Impurity model}

First we analyze the weight distribution in the ground state of the doped $\left(\mathrm{NiO}_{2}\right)^{2-}$ cluster with two holes per unit cell in $\mathrm{Ni}\left(3 d^{10}\right)$ and $\mathrm{O}\left(2 p^{6}\right)$ states, i.e., for the filling by $N_{\uparrow}=N_{\downarrow}=1$ hole. In Fig. 2(a) we see a rapid change from the singlet $(S=0)$ to triplet $(S=1)$ state at $\Delta_{c} \simeq 7.7 \mathrm{eV}$. This value is somewhat lower than $\Delta_{c}=8.1 \mathrm{eV}$ given in Ref. [12] as we use here a somewhat smaller hybridization; see Table I. However, overall we find a very good qualitative agreement with a distinct transition from the ZR-singlet state dominated by the $b_{1} L_{b_{1}}$ configuration to the $a_{1} b_{1}$ state, where both holes are mainly at the $\mathrm{Ni}$ ion in the high-spin configuration. We observe that this transition is governed by the interplay between the $d-p$ hybridization and Hund's exchange at the $\mathrm{Ni}$ ion, $J_{H}$.

The ground state for $\Delta<\Delta_{c}$ consists of one hole occupying the $|(\bar{z})\rangle$ and the oxygen state with the same symmetry
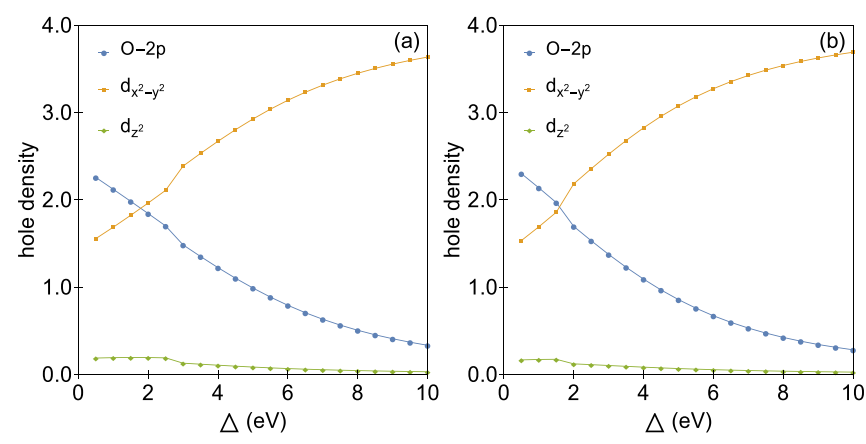

FIG. 3. Hole densities as obtained in the undoped system $\mathrm{Ni}_{4} \mathrm{O}_{8}$ of Fig. 1, $N_{\uparrow}=N_{\downarrow}=2$ holes, for increasing charge-transfer energy $\Delta$ and for (a) $U_{d p}=0$ and (b) $U_{d p}=1.0 \mathrm{eV}$. Different lines show the hole densities in different orbitals: oxygen $2 p$ orbitals (blue), $d_{x^{2}-y^{2}}$ orbital (orange), and $d_{z^{2}}$ orbital (green).

constructed out of four bonding $\left\{p_{i \sigma}^{\dagger}|0\rangle\right\}$ states, i.e.,

$$
p_{m \sigma}^{\dagger}=\frac{1}{2}\left(p_{1 \sigma}^{\dagger}-p_{2 \sigma}^{\dagger}-p_{3 \sigma}^{\dagger}+p_{4 \sigma}^{\dagger}\right),
$$

and the ZR singlet at site $m$ is

$$
\left|\phi_{m}\right\rangle=\frac{1}{\sqrt{2}}\left(d_{m(\bar{z}) \uparrow}^{\dagger} p_{m \downarrow}^{\dagger}-d_{m(\bar{z}) \downarrow}^{\dagger} p_{m \uparrow}^{\dagger}\right)|0\rangle .
$$

The weight of the ZR singlet is shown in Fig. 2 and labeled as $b_{1} L_{b_{1}}$. At $\Delta>\Delta_{c}$ the above weight becomes negligible, and the largest weight is found instead for the $\left|\psi_{m}\right\rangle=\left|a_{1} b_{1}\right\rangle$ state, which is antisymmetric for spins in $\{(z),(\bar{z})\}$ orbitals and labeled as $a_{1} b_{1}$.

Increasing intersite Coulomb repulsion to $U_{d p}=1 \mathrm{eV}$ moves the above transition to a value of $\Delta_{c}$ roughly lowered by $U_{d p}$, in agreement with the expectation from the mean-field approximation. Otherwise the weights of different configurations are almost the same, so one finds that this intersite charge-charge repulsion influences the energies of the two competing states and not their internal structure.

\section{B. Model of the $\mathrm{NiO}_{2}$ plane}

We next consider a cluster of $2 \times 2$ unit cells (each containing one $\mathrm{Ni}$ and two $\mathrm{O}$ ions) with periodic boundary conditions. The undoped system corresponds then to one hole per $\mathrm{Ni}$, i.e., to two holes with spin up and down, $N_{\uparrow}=N_{\downarrow}=2$ holes within a $\mathrm{Ni}_{4} \mathrm{O}_{8}$ cluster. Orbital-resolved densities are shown in Fig. 3 and go from an almost even distribution between $x^{2}-y^{2}$ and $2 p$ orbitals at small $\Delta$ to nearly complete localization on $x^{2}-y^{2}$ at large $\Delta$. Since there is on average just one hole per one $\mathrm{NiO}_{2}$ unit cell, there are only very few configurations where two holes may interact by $U_{d p}$, and this parameter does almost not change the ground state at $U_{d p}=0$; cf. Figs. 3(a) and 3(b).

Consider first the undoped system, i.e., the one with one hole per $\mathrm{NiO}_{2}$ unit cell. The hole is then predominantly in the orbital of $x^{2}-y^{2}$ symmetry. The weights of these configurations increase with increasing charge-transfer energy $\Delta$ when the holes hybridize more weakly with the surrounding oxygens. The many-hole wave function may be written 
here as

$$
\begin{aligned}
\left|\Phi_{22}\right\rangle= & \left\{a_{(\bar{z})(\bar{z})(\bar{z})(\bar{z})} \Pi_{m \in K_{\uparrow}} d_{m(\bar{z}) \uparrow}^{\dagger} \Pi_{n \in L_{\downarrow}} d_{n(\bar{z}) \downarrow}^{\dagger}+\cdots\right. \\
& \left.+b_{(z)(z)(z)(z)} \Pi_{m \in K_{\uparrow}} d_{m(z) \uparrow}^{\dagger} \Pi_{n \in L_{\downarrow}} d_{n(z) \downarrow}^{\dagger}+\cdots\right\}|0\rangle,
\end{aligned}
$$

where $K_{\sigma}$ and $L_{\sigma}$ are the sets of Ni ions which are occupied by $\sigma$-spin electrons and $a_{(\bar{z})(\bar{z})(\bar{z})(\bar{z})}$ and $b_{(z)(z)(z)(z)}$ are the coefficients of two competing states. For large charge-transfer energy $\Delta>6 \mathrm{eV}$ the holes concentrate within the $\left|x^{2}-y^{2}\right\rangle$ state at each $\mathrm{Ni}$ site in the undoped system. The hole density for the second $e_{g}$ orbital almost vanishes, and the high-spin states play no role.

Going to a doped system, there are many more configurations in the real space, and a very complex wave function similar to Eq. (11) will arise. Clearly, specifying the hole configurations in real space is unpractical. Therefore we shall focus attention on the leading two-hole configurations, such as those considered for an isolated $\mathrm{NiO}_{4}$ cluster; see Fig. 2 . Thus for a total of five holes in the $\mathrm{Ni}_{4} \mathrm{O}_{8}$ cluster, $N_{\uparrow}=3$ and $N_{\downarrow}=2$, we shall have components in the total wave function which have two holes in the states centered at one Ni site and otherwise one hole for the other three Ni sites. For the particular Ni site $m$ with two holes we shall write approximately the function centered at this site $m$ and two holes with opposite spins in both components,

$$
\begin{aligned}
\left|\Phi_{m}\right\rangle \approx & a_{m} \frac{1}{\sqrt{2}}\left(d_{m(\bar{z}) \uparrow}^{\dagger} p_{m 0 \downarrow}^{\dagger}-d_{m(\bar{z}) \downarrow}^{\dagger} p_{m 0 \uparrow}^{\dagger}\right)|0\rangle \\
& +b_{m} \frac{1}{\sqrt{2}}\left(d_{m(z) \uparrow}^{\dagger} d_{m(\bar{z}) \downarrow}^{\dagger}+d_{m(z) \downarrow}^{\dagger} d_{m(\bar{z}) \uparrow}^{\dagger}\right)|0\rangle .
\end{aligned}
$$

Here, the first line is the ZR singlet, the second line stands for the $\left|a_{1} b_{1}\right\rangle$ state considered before in Sec. IV A, and $\left\{a_{m}, b_{m}\right\}$ are the probability amplitudes of each of the elemental configurations. Of course, the ground state is more complex and also includes other configurations. Taking $\left|a_{m}\right|^{2}$ and $\left|b_{m}\right|^{2}$ as the respective probabilities, we obtain the main local configurations, with their weights $\left\{b_{1} L_{b_{1}}, a_{1} b_{1}\right\}$ displayed in Fig. 4. The finite weights of other configurations displayed as well in Fig. 4 are obtained in a similar way numerically and demonstrate the delocalization of holes over oxygens in the regime of small $\Delta$. Analyzing these weight distributions shows that the character of the wave function changes when the chargetransfer energy increases from small to large $\Delta$.

Figure 4 refers to the lowest doping achievable on a cluster with four unit cells, namely, one additional hole, i.e., $x=\frac{1}{4}$ doped hole per Ni site, or $N_{\uparrow}=3$ and $N_{\downarrow}=2$. In contrast to the single-Ni-ion results shown in Fig. 2, we no longer see a sharp jump, but rather a gradual transition. This is due to the transition from a rotationally invariant impurity to a translationally invariant lattice, where states of, e.g., $b_{1}$ and $a_{1}$ symmetry are allowed to hybridize. In agreement with this wave function, we observe that the most important two configurations for the ground state are $\left|b_{1} L_{b_{1}}\right\rangle$ and $\left|a_{1} b_{1}\right\rangle$ states. They stand for the ZR-singlet state and for the high-spin $S=1$ state stabilized by Hund's exchange when $\Delta>\Delta_{c}$. As observed for the impurity model in Fig. 2 and as expected, charge-transfer energies $\Delta \lesssim 7.0 \mathrm{eV}$ lead to a large weight in the ZR-singlet state, while the triplet state localized at $\mathrm{Ni}$
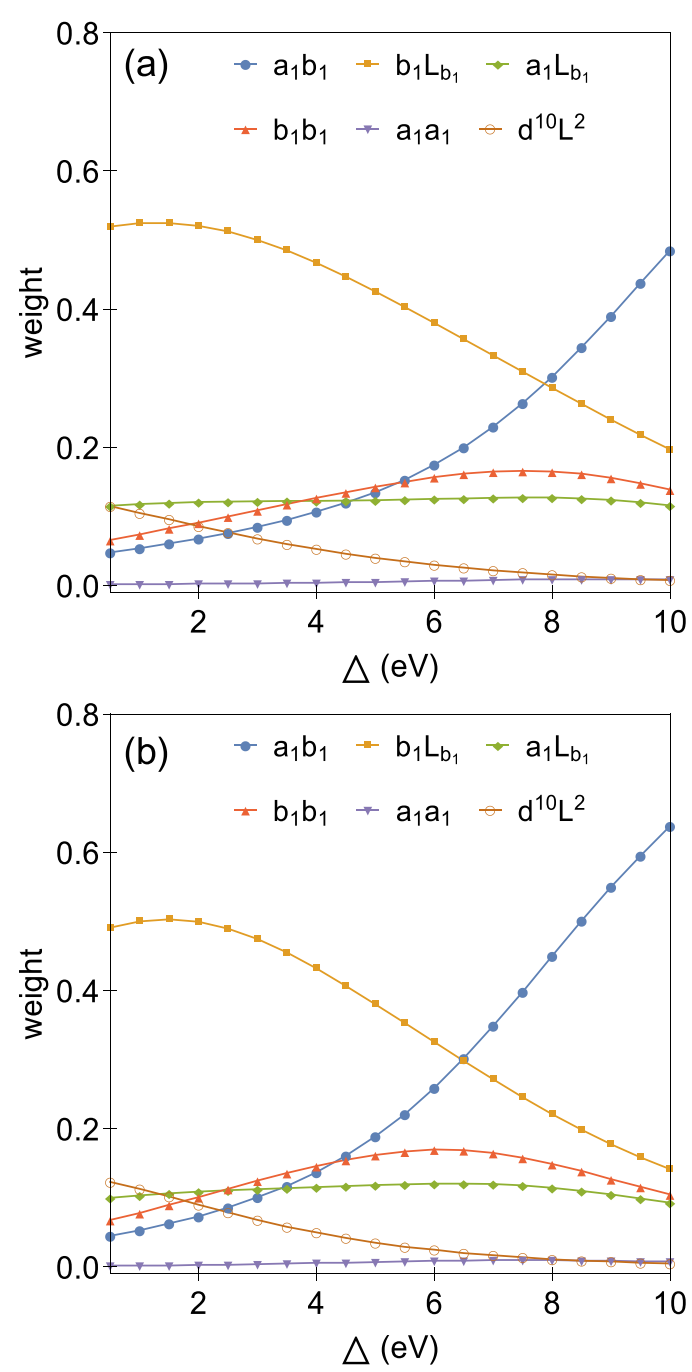

FIG. 4. The weights of various components (see legends) in the ground state of the $\mathrm{Ni}_{4} \mathrm{O}_{8}$ cluster shown in Fig. 1 as obtained for increasing $\Delta, N_{\uparrow}=3$, and $N_{\downarrow}=2$ for (a) $U_{d p}=0$ and (b) $U_{d p}=1.0 \mathrm{eV}$. The crossing point between the two ground-state components with the largest weights, $b_{1} L_{b_{1}}$ and $a_{1} b_{1}$, defines the crossover point between the two types of the ground state. It is moved to a smaller value of $\Delta$ when $U_{d p}=1.0 \mathrm{eV}$.

dominates at large $\Delta$. Again, nonlocal Coulomb repulsion $U_{d p}$ shifts this transition precisely in the range of $\Delta \approx 6-8 \mathrm{eV}$ expected to apply to nickelates, with $U_{d p}>0$ favoring the local triplet state already at lower crystal fields.

As already apparent in the impurity model in Fig. 2, other states in addition to the "pure" ZR-singlet and on-site $S=1$ states and more wave functions contribute, especially around the critical $\Delta \approx 7.0 \mathrm{eV}$. For instance, a "ZR" variant of the high-spin state contributes, where both holes have parallel spins (or are the third component of the triplet), but one hole is located on the $2 p$ orbitals. This state has rather constant weight over the whole range of $\Delta$, so that some $3 z^{2}-r^{2}$ character is expected for holes doped even into a cuprate regime with $\Delta=3.0 \mathrm{eV}$. This can be understood as being due to delocalization of the $2 p$ hole over adjacent $3 z^{2}-r^{2}$ orbitals. Conversely, doubly occupied $x^{2}-y^{2}$ orbitals also have a relevant weight for intermediate crystal fields $4.0 \lesssim \Delta \lesssim 10.0 \mathrm{eV}$. 

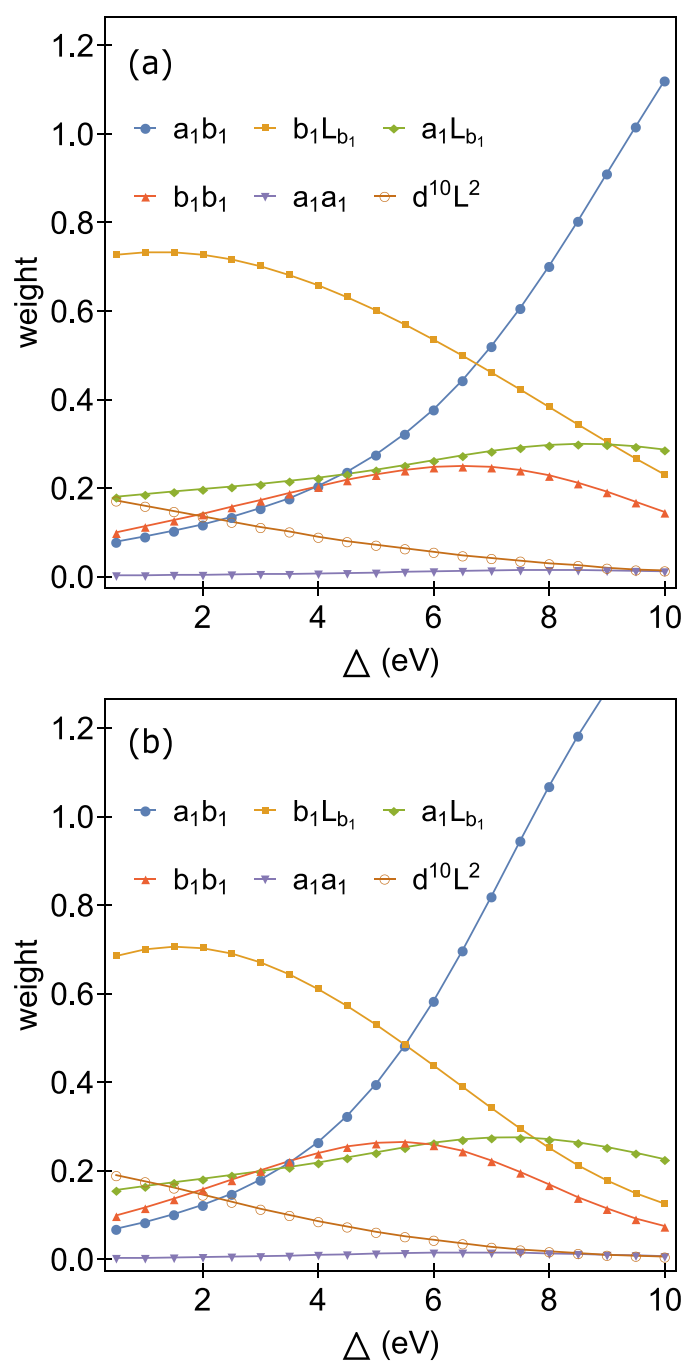

FIG. 5. The weights of the most important components in the ground state of the $\mathrm{Ni}_{4} \mathrm{O}_{8}$ cluster shown in Fig. 1 with one or two holes at Ni sites, as obtained for increasing $\Delta, N_{\uparrow}=N_{\downarrow}=3$, and (a) $U_{d p}=0$ and (b) $U_{d p}=1.0 \mathrm{eV}$.

For a system still in the regime of predominant singlets $(\Delta \lesssim 7.0 \mathrm{eV})$, the wave function is thus not necessarily a ZR singlet familiar from cuprates, where one hole has clear $2 p$ character, but can already have substantial "Mott" character with partly double occupied $x^{2}-y^{2}$ orbitals.

When two holes are added to the $\mathrm{Ni}_{4} \mathrm{O}_{8}$ cluster, i.e., $N_{\uparrow}=N_{\downarrow}=3$, one finds locally once again the low-spin ground state with ligand holes $\left|b_{1} L_{b_{1}}\right\rangle$ for small values of $\Delta<5 \mathrm{eV}$; see Fig. 5. This regime corresponds to doped cuprates. For even smaller values of $\Delta$ one finds stronger delocalization of holes onto the oxygen orbitals seen by the component of the $\left|d^{10} L^{2}\right\rangle$ state. However, for $\Delta \simeq 7 \mathrm{eV}$ as in nickelates [12], this state plays no role, and the ground state is qualitatively different-it has the largest weight for the high-spin state at Ni sites, $\left|a_{1} b_{1}\right\rangle$. This trend is enhanced by the realistic finite value of $U_{d p}=1.0 \mathrm{eV}$; cf. Figs. 5(a) and 5(b).

The evolution of the ground state in the doped systems may be qualitatively characterized by the transition from the

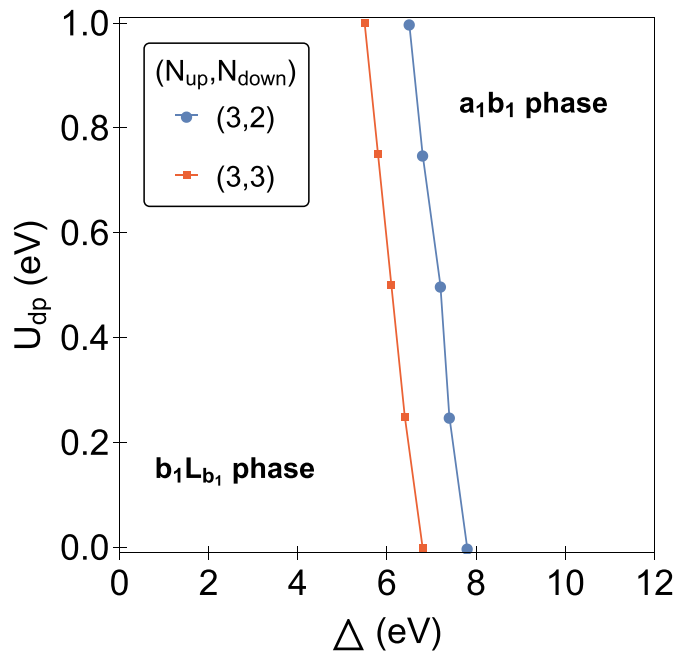

FIG. 6. Phase diagram arising from comparing the weight of the two dominant ground-state components for different values of $\Delta$ and $U_{d p}$. The blue line is for the $\mathrm{Ni}_{4} \mathrm{O}_{8}$ cluster doped by one hole $\left(N_{\uparrow}=3, N_{\downarrow}=2\right)$, and the red line is for the $\mathrm{Ni}_{4} \mathrm{O}_{8}$ cluster doped by two holes $\left(N_{\uparrow}=N_{\downarrow}=3\right)$.

ground state dominated by the low-spin $\left|b_{1} L_{b_{1}}\right\rangle$ local states to the more-localized-at-Ni-sites high-spin $\left|a_{1} b_{1}\right\rangle$ states, as shown in Fig. 6. The delocalization over oxygen orbitals occurs more easily in the low doping regime $x=\frac{1}{4}$, while for higher doping of $x=\frac{1}{2}$ the high-spin components dominate already for $\Delta<6.0 \mathrm{eV}$, taking the realistic finite value of $U_{d p}=1.0 \mathrm{eV}$.

\section{Discussion: Comparison between the $\mathrm{CuO}_{2}$ and $\mathrm{NiO}_{2}$ planes}

To illustrate the nature of the electronic states, we now consider the occupied and empty hole states. As the $\left(\mathrm{NiO}_{2}\right)^{3-}$ plane is negatively charged, it is more convenient to use here the hole notation; that is, the occupied states at low energy in the lower Hubbard band (LHB) are the states occupied by holes. In the undoped systems this means the filling of one hole per each $\mathrm{NiO}_{2}$ unit. First we consider such a system and show that the undoped $\mathrm{NiO}_{2}$ plane is insulating; see Fig. 7. Whether or not this corresponds to the real situation in $\mathrm{NdNiO}_{2}$ is an open question-we suggest that this system is in a poor metallic state after considerable self-doping. It increases the hole concentration in $\mathrm{NiO}_{2}$ planes beyond one hole per $\mathrm{NiO}_{2}$ unit cell when $\mathrm{Nd}$ ions have a smaller positive change than $\mathrm{Nd}^{3+}$.

We emphasize that due to the large value of $\Delta$ [12], nickelates are in the Mott-Hubbard regime of the ZaanenSawatzky-Allen diagram of correlated insulators [43]. This is also illustrated by two Hubbard bands: the LHB and the upper Hubbard band (UHB), separated by the large Mott-Hubbard gap in the undoped system (see Fig. 7). The LHB and the UHB have mainly the states at $\mathrm{Ni}$ sites, with little oxygen admixture. The ground state contains predominantly the holes within the $x^{2}-y^{2}$ orbitals in the LHB, while the $z^{2}$ states have a large weight for the lower edge of the UHB. This weight is enhanced for $U_{d p}=1.0 \mathrm{eV}$; cf. Figs. 7(a) and 7(b). Moreover, the character of the lowest UHB states changes with $U_{d p}$, 

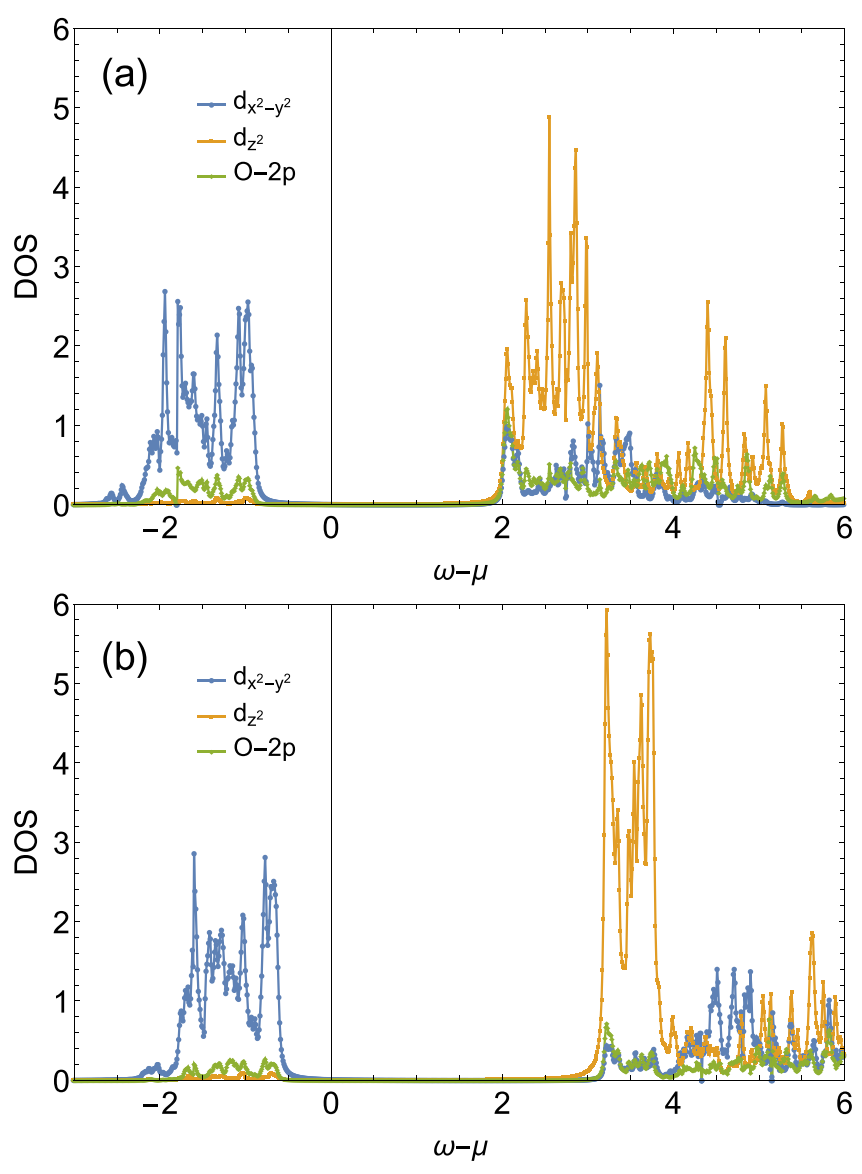

FIG. 7. Density of states (DOS) for the reference undoped $\mathrm{NiO}_{2}$ plane obtained via VCA and resolved for each orbital (blue, $d_{x^{2}-y^{2}}$ orbital; orange, $d_{z^{2}}$ orbital; green, oxygen $2 p$ orbital), for $\Delta=7.0 \mathrm{eV}$ and for (a) $U_{d p}=0$ and (b) $U_{d p}=1.0 \mathrm{eV}$; the other parameters are given in Table I. The LHB at negative energies is predominantly occupied by holes in $x^{2}-y^{2}$ orbitals. In the UHB the occupation of $z^{2}$ states is enhanced while that of oxygen states is suppressed with increasing $U_{d p}$.

going from a somewhat ZR-singlet-like band (albeit with $z^{2}$ admixture and reduced $2 p$ content) to an almost pure $z^{2}$ band.

To illustrate this point, let us first go back to the the ZR-singlet states arising in an analogous model applied to cuprates [42]. With a lower value of $\Delta=3.0 \mathrm{eV}$ [42], the $\mathrm{CuO}_{2}$ plane is in the charge-transfer regime. Hybridization between $x^{2}-y^{2}$ orbitals with oxygen $2 p$ states is here naturally stronger, so that the oxygen content of occupied states rises. In the electronic structure around the Fermi energy, shown in Fig. 8, one finds the lower part of the UHB to be dominated by $x^{2}-y^{2}$ states. Nevertheless, substantial oxygen- $p$ character is present, especially around $(\pi / 2, \pi / 2)$ at the very lowest edges of the spectrum.

When $U_{d p}$ is increased from 0 to $1 \mathrm{eV}$, the gap increases and orbital contributions to the bands are affected, but overall band shapes and their order remain the same; see Fig. 8(b). For instance, one finds slight changes in the orbital character of the lowest unoccupied states: In addition to $x^{2}-y^{2}$ and $2 p$ orbitals, even some $z^{2}$ weight is now present, especially around $(0, \pi)$ and $(\pi, 0)$. As can be seen in Fig. 4, hole doping

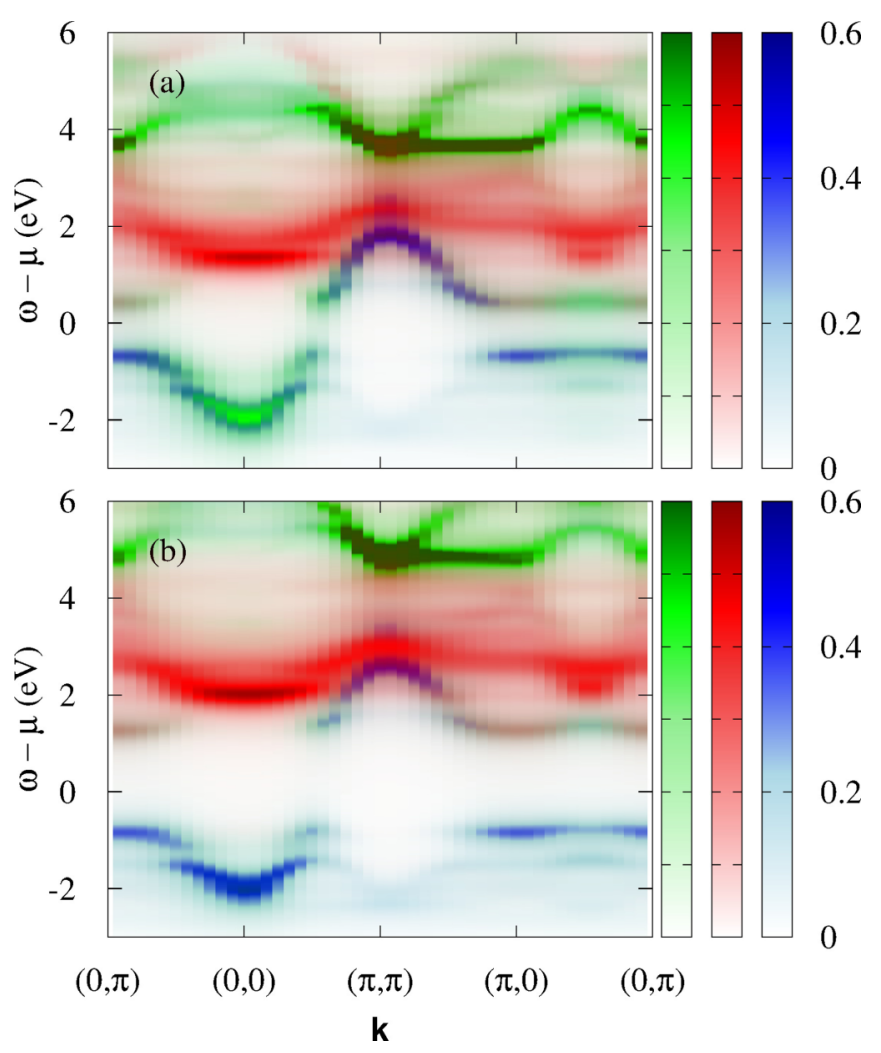

FIG. 8. The orbital-resolved and k-resolved spectra for the cuprate parameters with $\Delta=3.0 \mathrm{eV}$ along the high-symmetry lines in the $2 \mathrm{D}$ Brillouin zone for two values of $U_{d p}$ : (a) $U_{d p}=0$ and (b) $U_{d p}=1.0 \mathrm{eV}$. The other parameters are as in Table I. Blue and red shadings stand for $x^{2}-y^{2}$ and $z^{2}$ orbital states at $\mathrm{Ni}$ ions, while green shading corresponds to oxygen states.

only induces a very small triplet component for these parameters. The $z^{2}$ weight seen in the spectra is thus more easily understood as arising from delocalization of the ligand hole onto neighboring $z^{2}$ orbitals. When $U_{d p}$ becomes relevant, it pushes more of the ligand holes into $z^{2}$ states. Despite this modification of orbital makeup, the lowest states of the UHB are naturally explained in terms of a single ZR-singlet band for both $U_{d p}=0$ and $1 \mathrm{eV}$, while a $z^{2}$ band with a minimum at $(0,0)$ comes at higher energy.

Let us now come back to the nickelate regime with a larger $\Delta=7.0 \mathrm{eV}$, for which $\mathbf{k}$-resolved spectra are shown in Fig. 9; they correspond to the density of states and the Hubbard subbands of Fig. 7. At first sight, one immediately sees the stronger $z^{2}$ character near the lower edge of the UHB, both without and with $U_{d p}$. The main oxygen bands, on the other hand, are pushed to even higher energies outside the the energy window depicted. The usual ZR-singlet picture thus does not apply, as one can also infer from the sizable triplet component seen for $\Delta=7.0 \mathrm{eV}$ in Fig. 4.

For $U_{d p}=0$, however, the density of states in Fig. 7(a) clearly also shows some $2 p$ and $x^{2}-y^{2}$ weight in the first hole-doping states. The spectrum in Fig. 9(a) suggests the corresponding lowest unoccupied band to be related to the ZR-singlet band of the cuprate scenario in Fig. 8(b). The shape is similar, with more pronounced minima at $(\pi, 0)$ and $(0, \pi)$, but the orbital makeup differs from cuprates, with much 


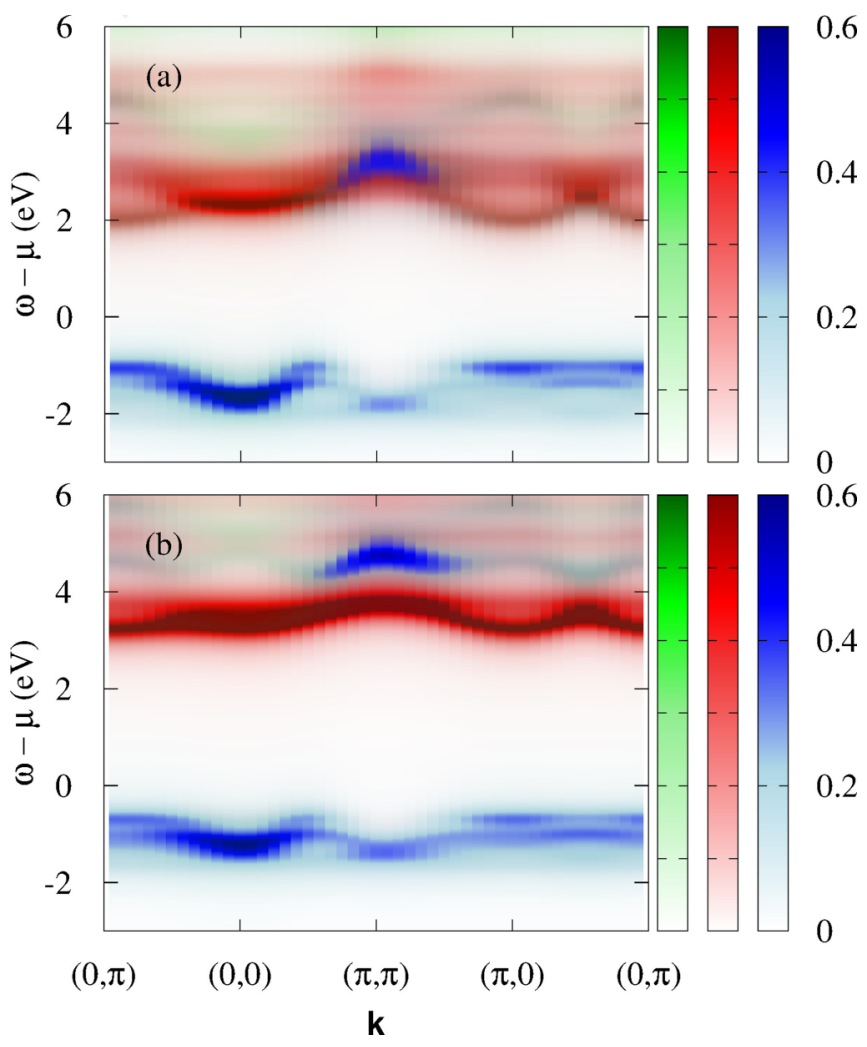

FIG. 9. The orbital and k-resolved spectra for the nickelate parameters (Table I) along the high-symmetry lines in the 2D Brillouin zone for (a) $U_{d p}=0.0 \mathrm{eV}$ and (b) $U_{d p}=1.0 \mathrm{eV}$; the other parameters are as in Table I. Blue and red shadings stand for $x^{2}-y^{2}$ and $z^{2}$ states at $\mathrm{Ni}$ ions, while green shading corresponds to oxygen states.

reduced $2 p$ and much increased $z^{2}$ character. A description of the lowest states in terms of a ZR-singlet-like band appears still plausible, even though the different orbital makeup and the resulting substantial triplet admixture suggest that effective interaction parameters can be quite different from those describing hole doping in cuprates.

At $U_{d p}=1 \mathrm{eV}$, finally, both the density of states in Fig. 7(b) and the k-resolved spectrum in Fig. 9(b) show the lowest unoccupied states to be of almost pure $z^{2}$ character. While some $2 p$ and $x^{2}-y^{2}$ character is still present, the system is now more easily understood as a "classical" MottHubbard insulator, where the gap separates Hubbard subbands built mainly by the orbital states at $\mathrm{Ni}$ ions. Accordingly, the on-site triplet dominates over the ZR singlet in Fig. 4.

\section{SUMMARY}

In summary, we have investigated the ground state of $\mathrm{NiO}_{2}$ planes in nickelate superconductors at increasing hole doping. First of all, we find that the local high-spin states $\left|a_{1} b_{1}\right\rangle$ are important for $\Delta \simeq 7.0 \mathrm{eV}$, as expected for nickelate superconductors. However, there is a crossover transition between the two regimes, dominated by low-spin and high-spin states, when the charge-transfer energy increases.

Second, we would like to point out that $U_{d p}$ is an important parameter to model the situation in doped $\mathrm{NiO}_{2}$ planes. The Coulomb repulsion between $\mathrm{Ni}$ and $\mathrm{O}$ sites influences the stability of low-spin ZR states as this interaction is to a large extent missing in the competing high-spin states. The value of $\Delta \simeq 7.0 \mathrm{eV}$ is in the critical regime where the weight distribution and the nature of the ground state depend in a subtle way on the value of $\Delta$.

Finally, as our third conclusion we wish to point out the increasing admixture of high-spin states at $\mathrm{Ni}$ ions with increasing charge-transfer energy $\Delta$ in the ground state of doped nickelates. We have shown that the $z^{2}$ orbitals are the next to be occupied by doped holes in case any extra splitting between the $z^{2}$ and the $x^{2}-y^{2}$ orbitals is absent. In this way the holes concentrate at $\mathrm{Ni}$ sites, and the population of the $S=1$ states is increased. In nickelate films the orbitals which are preferentially occupied are instead $x y$, but they lead to the same global result that high-spin states arise locally. In the Appendix we present arguments as to how the upper Hubbard band may change when the $x y$ orbitals are filled instead by holes rather than the $z^{2}$ ones.

In the absence of an electrostatic crystal field, the hybridization with $2 p$ orbitals indeed slightly favors the $z^{2}$ orbitals over the $t_{2 g}$ states, but the difference is not large. Electrostatic crystal fields due to the planar geometry and missing apical oxygens can thus easily tip the balance. However, the emerging scenario remains equivalent: At $\Delta \approx 7.0 \mathrm{eV}$ and $U_{d p}=0$, hole doping still leads to large singlet weight, while triplet states (now involving the $x y$ orbitals) dominate for $U_{d p}=1 \mathrm{eV}$.

In general, we find that hole doping distributes over the entire system, in contrast to the onset of local $S=1$ states [22]. This issue is, however, still open and should be investigated further. At the moment we can conclude that the contribution of high-spin states to the electronic structure arises in any case, independently of which $\mathrm{Ni}$ orbitals are occupied by a second hole in doped systems.

Note added. Recently, it was established by local density approximation with $U$ method $(\mathrm{LDA}+U)$ that both $S=0$ and $S=1$ two-hole states arise by doping and compete in doped $\mathrm{NdNiO}_{2}$ [44]. As a result, the nature of the electronic structure and exchange interactions are three-dimensional. This observations should be important in future studies of nickelate superconductors.

\section{ACKNOWLEDGMENTS}

We thank Andres Greco, Krzysztof Rościszewski, and George A. Sawatzky for very insightful discussions. We thank Ali Alavi for informing us about the content of Ref. [26] prior to publication. This research was supported in part by the National Science Foundation under Grant No. NSF PHY-1748958. T.P. acknowledges the Development and Promotion of Science and Technology Talents Project (DPST). A.M.O. acknowledges Narodowe Centrum Nauki (NCN, Poland) Project No. 2016/23/B/ST3/00839 and is grateful for support from the Alexander von Humboldt Foundation Fellowship (Humboldt-Forschungspreis).

\section{APPENDIX: THE MODEL WITH $x y$ ORBITALS}

There is some discussion concerning the order of the $d$ orbitals, apart from the commonly accepted view that holes 


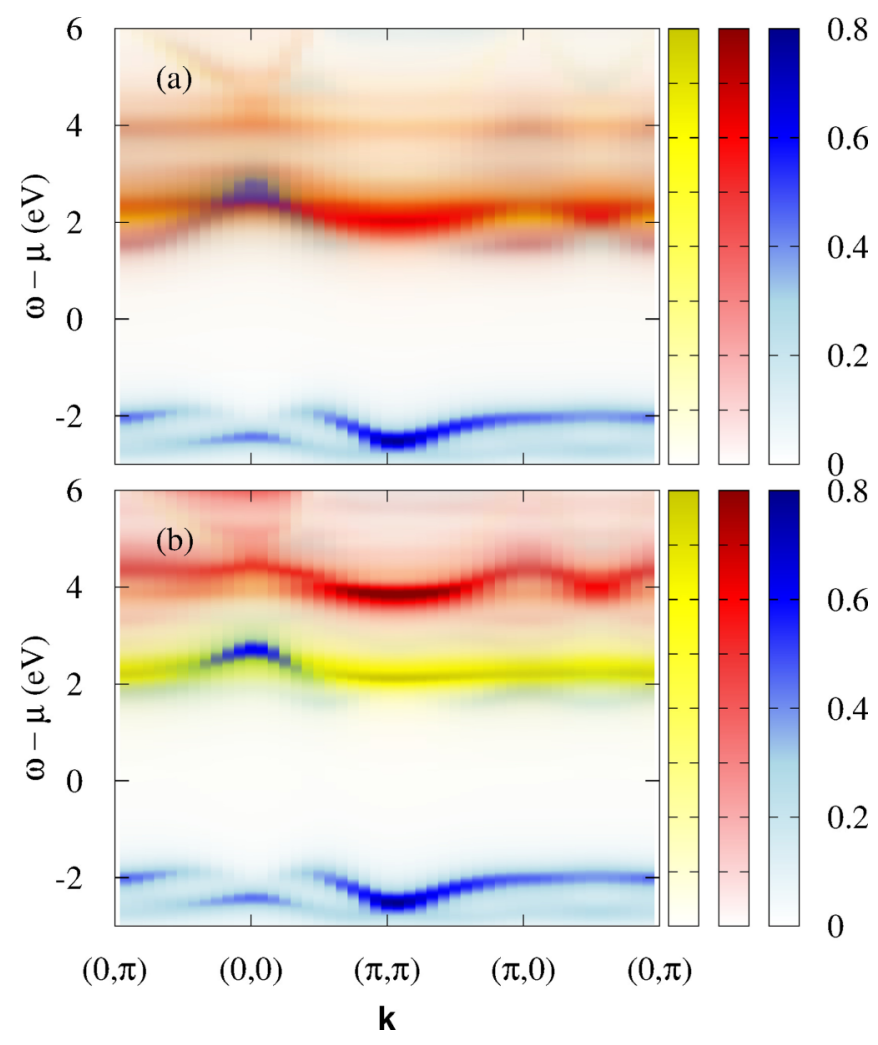

FIG. 10. k-resolved one-particle spectra obtained for the sevenorbital model which includes three $\left\{x^{2}-y^{2}, 3 z^{2}-r^{2}, x y\right\}$ orbitals per Ni site and two $\left\{p_{x}, p_{y}\right\}$ orbitals at oxygen sites in the $\mathrm{NiO}_{2}$ unit cell of Fig. 1. In (a) there is no explicit crystal field between the $x^{2}-y^{2}$ and $3 z^{2}-r^{2}$ orbitals (5), so that the $x y$ orbital lies slightly above the lowest $3 z^{2}-r^{2}$ states. In (b) the crystal field $\Delta_{z^{2}}=2.0 \mathrm{eV}$ [see Eq. (5)] raises the $3 z^{2}-r^{2}$ orbital, but the basic scenario of holes entering a ZR-singlet band remains intact. The parameters are as in Table I, and $\Delta=7.0 \mathrm{eV}, U_{p d}=0$. Yellow shading refers to the $x y$ orbitals, red refers to the $3 z^{2}-r^{2}$ orbitals, and blue refers to the $x^{2}-y^{2}$ orbitals.

in the "undoped" reference system are preferentially found in $x^{2}-y^{2}$ orbitals. Due to the planar cluster geometry (see Fig. 1), one can argue that the charge repulsion coming from the oxygen ions favors the electron occupation of the $3 z^{2}-r^{2}$ orbital and that the $x y$ orbital should come below the $x^{2}-y^{2}$ orbital, as indeed suggested by the crystal-field splittings [25]. Such arguments were also given, for instance, in Refs. [45,46] and are supported by quantum-chemistry calculations of hole levels [26].

On the other hand, one can argue that effective crystalfield splitting is often determined by hybridization rather than electrostatic forces. In this picture, putting a hole into the $3 z^{2}-r^{2}$ orbital is favorable because it can then delocalize better than the hole which resides in the $x y$ orbital. Such effects resulting in the level order opposite to the one expected from electrostatic charge have, e.g., been observed in iridates with their extended $5 d$ wave functions [47]. In the $a$ (b) direction, the corresponding hopping parameters are $t_{p d} / 2 \simeq \sqrt{3} / 2 \simeq 0.866 \mathrm{eV}$ for the hopping between the $3 z^{2}-r^{2}$ orbital and the $p_{x}\left(p_{y}\right)$ orbital vs $(p d \pi) \simeq 0.75 \mathrm{eV}$ for hopping between $x y$ and $p_{y}\left(p_{y}\right)$. Based on the analysis of

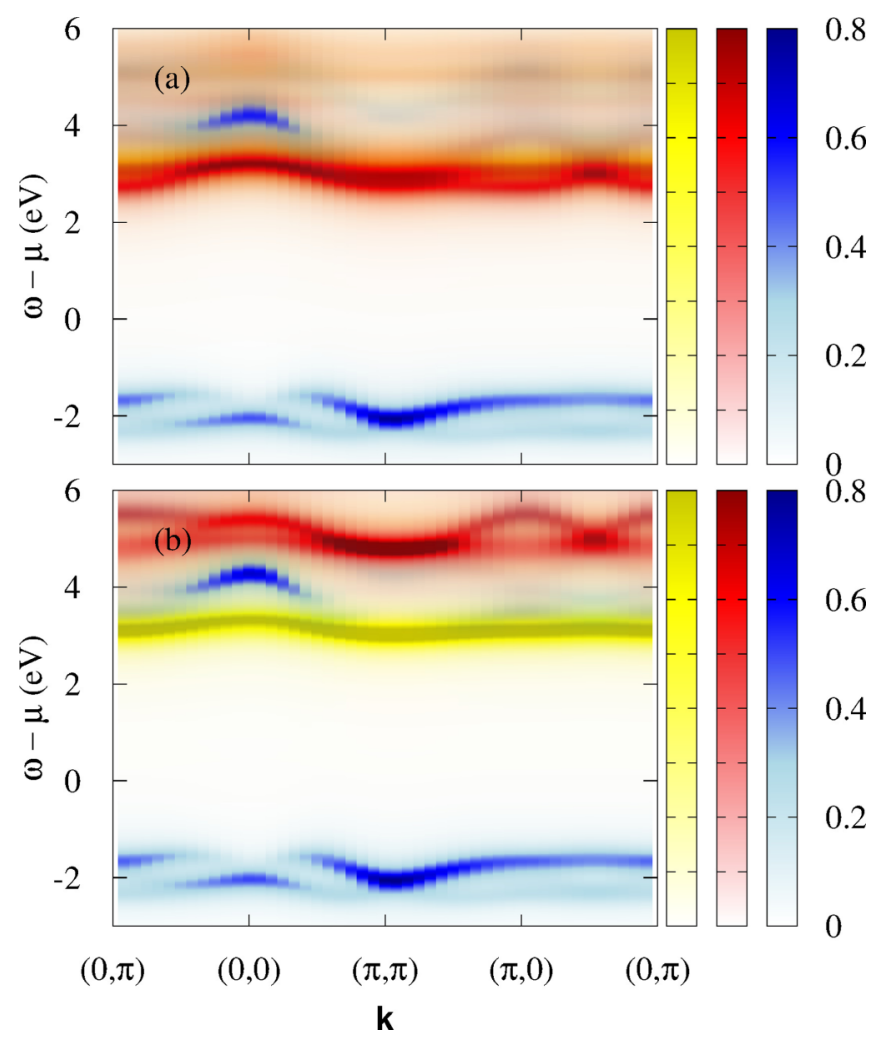

FIG. 11. As in Fig. 10, but for $U_{p d}=1.0 \mathrm{eV}$. In (a) without a crystal field, the lowest hole-doped states are now $3 z^{2}-r^{2}$, while they have $x y$ character when $\Delta_{z^{2}}=2.0 \mathrm{eV}$ (5) in (b). The color convention is the same as in Fig. 10.

Ref. [48], where hybridization effects are found to dominate over point-charge effects, it has been argued [12] that the $3 z^{2}-r^{2}$ orbital should be considered preferentially.

A closer look at the results reported in Ref. [48] indicates, however, that the hybridization effects are not very large and in fact the strongest in the "charge-transfer" limit of a smaller crystal field separating oxygen and the transition-metal ion. When the hole becomes more localized onto the transitionmetal ion, the impact of hybridization with oxygen is reduced, so that the energy difference between $x y$ and $3 z^{2}-r^{2}$ shrinks. We thus investigate the aspect of level ordering in this Appendix.

To do so, we extend the model to include three $3 d$ orbitals $\left\{x^{2}-y^{2}, 3 z^{2}-r^{2}, x y\right\}$ per each Ni ion as well as two $\left\{p_{x}, p_{y}\right\}$ at each oxygen and study it using the VCA. We then have to truncate the Hilbert space of the four-unit-cell cluster but wish to point out that a three-unit-cell cluster with the full Hilbert space gave equivalent results. Since the $x^{2}-y^{2}$ orbital was nearly always found to be half filled, we restrict possible states to those with at least two holes in these orbitals in the cluster shown in Fig. 1. In other words, we allow at most two of the four $x^{2}-y^{2}$ orbitals to be completely filled.

Parameters referring to the orbitals included in the model with a smaller basis set of the main text, as summarized in Table I, are kept here. In addition, Hund's exchange coupling $J_{H}^{p}=0.8 \mathrm{eV}$ on oxygen ions as well as the corresponding interorbital $U_{p}^{\prime}=U_{p}-2 J_{H}^{p}$ was introduced. Additional hoppings were chosen following Ref. [48]: $t_{p p^{\prime}}=-0.35 \mathrm{eV}$ 
between nearest-neighbor parallel oxygen orbitals, and $t_{x y}=t_{p d} / 2=0.65 \mathrm{eV}$ connecting the $x y$ orbital to oxygen. For the signs resulting from orbital phases, see Ref. [48].

Without any static point-charge fields, the $x y$ orbital is indeed found to be above the $3 z^{2}-r^{2}$ orbital (in the hole notation), so that one would encounter the latter first when hole-doping the compound. Below we shall follow here the first-principles calculations which give the $x y$ orbitals as the energetically closest ones to $x^{2}-y^{2}$ [25]. If this is not assumed, for $U_{d p}=0$, holes enter first a band composed of a mixture of $x^{2}-y^{2}, 3 z^{2}-r^{2}$, and $2 p$ orbitals; see Fig. 10(a). $3 z^{2}-r^{2}$ states are mixed into this band, as seen before in Fig. 9(a), while $x y$ states come at slightly higher energies and do not mix noticeably with the lowest hole band.

However, the splitting between the $3 d$ states, $3 z^{2}-r^{2}$ and $x y$, is very small indeed, and moderate explicit crystal fields

$$
H_{\Delta_{z^{2}}}=\Delta_{z^{2}} \sum_{m} n_{m, z^{2}},
$$

with the sum going over all $\mathrm{Ni}$ ions and $n_{m, z^{2}}$ referring to the hole density in the $3 z^{2}-r^{2}$ orbital, could easily reverse their order. Such a situation is shown in Fig. 10(b) for a moderately large $\Delta_{z^{2}}=2.0 \mathrm{eV}$, which is in line with recent quantum-chemistry results [26]. Nevertheless, the conclusions from the main text remain valid: For $U_{d p}=0$, the lowest hole-doping states are composed of $x^{2}-y^{2}$ and $2 p$ states. In fact, their lower $3 z^{2}-r^{2}$ population and nearly absent $x y$ character make this band rather more similar to the ZR-singlet band known from cuprates.

Once $U_{d p}=1 \mathrm{eV}$, the Mott-Hubbard gap increases (Fig. 11), and doped holes hardly go into the $x^{2}-y^{2}$ orbital, regardless of whether $\Delta_{z^{2}}=0$ or $\Delta_{z^{2}}=2 \mathrm{eV}$. For $\Delta_{z^{2}}=0$, the lowest hole-doping states are now of almost exclusively $3 z^{2}-r^{2}$ character; see Fig. 11(a). Once $\Delta_{z^{2}}=2.0 \mathrm{eV}$, the crystal field pushes the $3 z^{2}-r^{2}$ states towards higher energies, and holes enter a nearly pure $x y$ band instead; see Fig. 11(b).

We thus conclude that the $x y$ orbital is likely to be relevant to doped $\mathrm{NiO}_{2}$ planes but that this does not affect our results: For $U_{p d}=0$, a band with robust $x^{2}-y^{2}$ orbital character hosts the lowest hole-doping states regardless of which orbital comes next; see Fig. 10. For $U_{p d}=1.0 \mathrm{eV}$, conversely, holes enter preferentially either the $x y$ or $3 z^{2}-r^{2}$ orbital, depending on their relative crystal field (see Fig. 11), and high-spin $S=1$ states form locally.

Since the $x y$ orbital bonds to the orbitals orthogonal to those coupling with both the $x^{2}-y^{2}$ and $z^{2}$ orbitals, $x^{2}-y^{2}$ states see less oxygen-mediated hybridization with $x y$ than with $z^{2}$ states. The ZR-singlet-like band thus remains more clearly separated from the $x y$ band than from the $3 z^{2}-r^{2}$ states. In the full system, however, the "other" orbitals in addition to $x^{2}-y^{2}$ have been shown to hybridize strongly with rare-earth states and also with each other [49]. This clear separation of $x y$ and $x^{2}-y^{2}$ orbitals may thus be an artifact of our model, while the stronger mixing of $x^{2}-y^{2}$ states with a second band-as discussed in the main text-may be more realistic [11].
[1] V. I. Anisimov, D. Bukhvalov, and T. M. Rice, Electronic structure of possible nickelate analogs to the cuprates, Phys. Rev. B 59, 7901 (1999).

[2] D. Li, K. Lee, B. Y. Wang, M. Osada, S. Crossley, H. R. Lee, Y. Cui, Y. Hikita, and H. Y. Hwang, Superconductivity in an infinite-layer nickelate, Nature (London) 572, 624 (2019).

[3] A. M. Oleś, K. Wohlfeld, and G. Khaliullin, Orbital symmetry and orbital excitations in high- $T_{c}$ superconductors, Condens. Matter 4, 46 (2019).

[4] P. Hansmann, X. Yang, A. Toschi, G. Khaliullin, O. K. Andersen, and K. Held, Turning a Nickelate Fermi Surface into a Cupratelike One through Heterostructuring, Phys. Rev. Lett. 103, 016401 (2009).

[5] A. S. Disa, D. P. Kumah, A. Malashevich, H. Chen, D. A. Arena, E. D. Specht, S. Ismail-Beigi, F. J. Walker, and C. H. Ahn, Orbital Engineering in Symmetry-Breaking Polar Heterostructures, Phys. Rev. Lett. 114, 026801 (2015).

[6] K. A. Müller and J. G. Bednorz, Possible high- $T_{c}$ superconductivity in the Ba-La-Cu-O system, Z. Phys. B: Condens. Matter 64, 189 (1986).

[7] P. A. Lee, N. Nagaosa, and X.-G. Wen, Doping a Mott insulator: Physics of high-temperature superconductivity, Rev. Mod. Phys. 78, 17 (2006).

[8] B. Keimer, S. A. Kivelson, M. R. Norman, S. Uchida, and J. Zaanen, High temperature superconductivity in the cuprates, Nature (London) 518, 179 (2015).
[9] Y.-H. Zhang and A. Vishwanath, Type-II $t-J$ model in superconducting nickelate $\mathrm{Nd}_{1-x} \mathrm{Sr}_{x} \mathrm{NiO}_{2}$, Phys. Rev. Research 2, 023112 (2020).

[10] S. Bandyopadhyay, P. Adhikary, T. Das, I. Dasgupta, and T. Saha-Dasgupta, Superconductivity in infinite-layer nickelates: Role of $f$ orbitals, Phys. Rev. B 102, 220502(R) (2020).

[11] M. Hepting, D. Li, C. J. Jia, H. Lu, E. Paris, Y. Tseng, X. Feng, M. Osada, E. Been, Y. Hikita, Y.-D. Chuang, Z. Hussain, K. J. Zhou, A. Nag, M. Garcia-Fernandez, M. Rossi, H. Y. Huang, D. J. Huang, Z. X. Shen, T. Schmitt et al., Electronic structure of the parent compound of superconducting infinite-layer nickelates, Nat. Mater. 19, 381 (2020).

[12] Mi Jiang, M. Berciu, and G. A. Sawatzky, Critical Nature of the Ni Spin State in Doped $\mathrm{NdNiO}_{2}$, Phys. Rev. Lett. 124, 207004 (2020).

[13] F. Lechermann, Late transition metal oxides with infinitelayer structure: Nickelates versus cuprates, Phys. Rev. B 101, 081110(R) (2020).

[14] F. Lechermann, Multiorbital Processes Rule the $\mathrm{Nd}_{1-x} \mathrm{Sr}_{x} \mathrm{NiO}_{2}$ Normal State, Phys. Rev. X 10, 041002 (2020).

[15] D. Li, B. Y. Wang, K. Lee, S. P. Harvey, M. Osada, B. H. Goodge, L. F. Kourkoutis, and H. Y. Hwang, Superconducting Dome in $\mathrm{Nd}_{1-x} \mathrm{Sr}_{x} \mathrm{NiO}_{2}$ Infinite Layer Films, Phys. Rev. Lett. 125, 027001 (2020).

[16] L. Si, W. Xiao, J. Kaufmann, J. M. Tomczak, Y. Lu, Z. Zhong, and K. Held, Topotactic Hydrogen in Nickelate Superconduc- 
tors and Akin Infinite-Layer Oxides $A B \mathrm{O}_{2}$, Phys. Rev. Lett. 124, 166402 (2020).

[17] P. Adhikary, S. Bandyopadhyay, T. Das, I. Dasgupta, and T. Saha-Dasgupta, Orbital-selective superconductivity in a twoband model of infinite-layer nickelates, Phys. Rev. B 102, 100501(R) (2020).

[18] B. Geisler and R. Pentcheva, Fundamental difference in the electronic reconstruction of infinite-layer versus perovskite neodymium nickelate films on $\mathrm{SrTiO}_{3}(001)$, Phys. Rev. B 102, 020502(R) (2020).

[19] M. Kitatani, L. Si, R. Arita, Z. Zhong, and K. Held, Nickelate superconductors - a renaissance of the one-band Hubbard model, npj Quantum Mater. 5, 59 (2020).

[20] A. S. Botana and M. R. Norman, Similarities and Differences between $\mathrm{LaNiO}_{2}$ and $\mathrm{CaCuO}_{2}$ and Implications for Superconductivity, Phys. Rev. X 10, 011024 (2020).

[21] F. Petocchi, V. Christiansson, F. Nilsson, F. Aryasetiawan, and P. Werner, Normal State of $\mathrm{Nd}_{1-x} \mathrm{Sr}_{x} \mathrm{NiO}_{2}$ from Self-Consistent $G W+$ EDMFT, Phys. Rev. X 10, 041047 (2020).

[22] J. Zaanen, A. M. Oleś, and P. Horsch, Generalizing the $t-J$ model: Triplet holes, Phys. Rev. B 46, 5798 (1992).

[23] F. C. Zhang and T. M. Rice, Effective Hamiltonian for the superconducting Cu oxides, Phys. Rev. B 37, 3759 (1988).

[24] I. Leonov, S. L. Skornyakov, and S. Y. Savrasov, Lifshitz transition and frustration of magnetic moments in infinitelayer $\mathrm{NdNiO}_{2}$ upon hole doping, Phys. Rev. B 101, 241108(R) (2020).

[25] X. Wu, D. Di Sante, T. Schwemmer, W. Hanke, H. Y. Hwang,

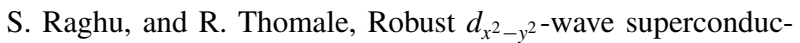
tivity of infinite-layer nickelates, Phys. Rev. B 101, 060504(R) (2020).

[26] V. M. Katukuri, N. A. Bogdanov, O. Weser, J. van den Brink, and A. Alavi, Electronic correlations and magnetic interactions in infinite-layer $\mathrm{NdNiO}_{2}$, Phys. Rev. B 102, 241112(R) (2020).

[27] K. Rościszewski and A. M. Oleś, Charge-transfer model for the electronic structure of layered ruthenates, Phys. Rev. B 91, 155137 (2015).

[28] J. Zaanen and A. M. Oleś, Canonical perturbation theory and the two-band model for high- $T_{c}$ superconductors, Phys. Rev. B 37, 9423 (1988).

[29] K. Rościszewski and A. M. Oleś, Spin-orbital order in $\mathrm{LaMnO}_{3}$ : $d-p$ model study, Phys. Rev. B 99, 155108 (2019).

[30] A. M. Oleś, Antiferromagnetism and correlation of electrons in transition metals, Phys. Rev. B 28, 327 (1983).

[31] S. Sugano, Y. Tanabe, and H. Kamimura, Multiplets of Transition Metal Ions in Crystals (Academic, New York, 1970).

[32] T. Mizokawa and A. Fujimori, Unrestricted Hartree-Fock study of transition-metal oxides: Spin and orbital ordering in perovskite-type lattice, Phys. Rev. B 51, 12880 (1995).

[33] T. Mizokawa and A. Fujimori, Electronic structure and orbital ordering in perovskite-type $3 d$ transition-metal oxides studied by Hartree-Fock band-structure calculations, Phys. Rev. B 54, 5368 (1996).
[34] J. C. Slater and G. F. Koster, Simplified LCAO method for the periodic potential problem, Phys. Rev. 94, 1498 (1954).

[35] E. Koch, The Lanczos method, in The LDA+DMFT Approach to Strongly Correlated Materials, edited by E. Pavarini, E. Koch, D. Vollhardt, and A. Lichtenstein (Forschungszentrum Jülich, Jülich, 2011), Chap. 8, pp. 8.1-8.30.

[36] M. Potthoff, M. Aichhorn, and C. Dahnken, Variational Cluster Approach to Correlated Electron Systems in Low Dimensions, Phys. Rev. Lett. 91, 206402 (2003).

[37] M. Potthoff, Self-energy-functional approach: Analytical results and the Mott-Hubbard transition, Eur. Phys. J. B 36, 335 (2003).

[38] M. Aichhorn and E. Arrigoni, Weak phase separation and the pseudogap in the electron-doped cuprates, EPL 72, 117 (2005).

[39] M. Aichhorn, H. G. Evertz, W. von der Linden, and M. Potthoff, Charge ordering in extended Hubbard models: Variational cluster approach, Phys. Rev. B 70, 235107 (2004).

[40] M. Daghofer and M. Hohenadler, Phases of correlated spinless fermions on the honeycomb lattice, Phys. Rev. B 89, 035103 (2014).

[41] F. Adler, S. Rachel, M. Laubach, J. Maklar, A. Fleszar, J. Schäfer, and R. Claessen, Correlation-Driven Charge Order in a Frustrated Two-Dimensional Atom Lattice, Phys. Rev. Lett. 123, 086401 (2019).

[42] E. Arrigoni, M. Aichhorn, M. Daghofer, and W. Hanke, Phase diagram and single-particle spectrum of $\mathrm{CuO}_{2}$ high- $T_{c}$ layers: Variational cluster approach to the three-band Hubbard model, New J. Phys. 11, 055066 (2009).

[43] J. Zaanen, G. A. Sawatzky, and J. W. Allen, Band Gaps and Electronic Structure of Transition-Metal Compounds, Phys. Rev. Lett. 55, 418 (1985).

[44] X. Wan, V. Ivanov, G. Resta, I. Leonov, and S. Y. Savrasov, Exchange interactions and sensitivity of the Ni two-hole spin state to Hund's coupling in doped $\mathrm{NdNiO}_{2}$, Phys. Rev. B 103, 075123 (2021).

[45] Y. Zhang, L.-F. Lin, W. Hu, A. Moreo, S. Dong, and E. Dagotto, Similarities and differences between nickelate and cuprate films grown on $\mathrm{SrTiO}_{3}$ substrate, Phys. Rev. B 102, 195117 (2020).

[46] M. Rossi, H. Lu, A. Nag, D. Li, M. Osada, K. Lee, B. Y. Wang, S. Agrestini, M. Garcia-Fernandez, Y.-D. Chuang, Z. X. Shen, H. Y. Hwang, B. Moritz, K.-J. Zhou, T. P. Devereaux, and W. S. Lee, Orbital and spin character of doped carriers in infinite-layer nickelates, arXiv:2011.00595.

[47] J. Kim, M. Daghofer, A. H. Said, T. Gog, J. van den Brink, G. Khaliullin, and B. J. Kim, Excitonic quasiparticles in a spinorbit Mott insulator, Nat. Commun. 5, 4453 (2014).

[48] M. Jiang, M. Moeller, M. Berciu, and G. A. Sawatzky, Relevance of $\mathrm{Cu}-3 d$ multiplet structure in models of high- $T_{c}$ cuprates, Phys. Rev. B 101, 035151 (2020).

[49] Y. Gu, S. Zhu, X. Wang, J. Hu, and H. Chen, A substantial hybridization between correlated Ni- $d$ orbital and itinerant electrons in infinite-layer nickelates, Commun. Phys. 3, 84 (2020). 